\title{
Rendimiento de la evaluación cefalométrica para el diagnóstico sagital intermaxilar. Revisión narrativa
}

\author{
Performance of the cephalometric asessment for sagital \\ intermaxillary diagnosis. A narrative review
}

\author{
Castro Arenas MV ${ }^{1}$, Hurtado Peralta $\mathrm{M}^{1}$, Oyonarte Weldt $\mathrm{R}^{2}$
}

\begin{abstract}
RESUMEN
Las anomalías dentomaxilares sagitales son entidades clínicas altamente prevalentes, que afectan entre un $20 \%$ y un $40 \%$ de la población; en un gran porcentaje se deben a problemas esqueletales máxilo-mandibulares, y se caracterizan por alteraciones del resalte incisivo. Su relevancia radica en que cuando sus manifestaciones son severas, éstas generan alteraciones morfológicas que traen consecuencias funcionales, estéticas y psicológicas, tanto en niños como en adultos. La evaluación cuantitativa de las relaciones sagitales intermaxilares se realiza preferentemente con cefalometría, a través de mediciones que constituyen indicadores diagnósticos. Existen numerosos índices para la clasificación sagital intermaxilar, sin embargo, la información respecto de su valor diagnóstico es escasa, en términos de la sensibilidad y especificidad asociadas al uso de ellos. La presente revisión narrativa tiene por objetivo analizar el rendimiento de la evaluación cefalométrica como herramienta diagnóstica para la medición de la relación sagital intermaxilar en términos de sensibilidad y especificidad. En la actualidad no existe una sola prueba que reúna todas las características ideales para determinar con precisión si un individuo presenta una alteración sagital esqueletal. La información disponible referente a las propiedades de especificidad y sensibilidad de las mediciones cefalométricas sagitales intermaxilares como indicadores diagnósticos es escasa. Entre ellas, el ángulo ANB constituye una herramienta adecuada, simple y válida para determinar estas desarmonías. El uso de pruebas diagnósticas cefalométricas para la evaluación sagital intermaxilar en individuos en crecimiento debe considerar la naturaleza dinámica del desarrollo craneofacial.
\end{abstract}

Rev. Clin. Periodoncia Implantol. Rehabil. Oral Vol. 6(2); 99-104, 2013.

Palabras clave: Cefalometría, diagnóstico sagital intermaxilar, sensibilidad, especificidad.

\begin{abstract}
Sagittal intermaxillary anomalies are highly prevalent clinical entities that affect between $20 \%$ and $40 \%$ of the population. The origin of a high percentage of them relies on skeletal alterations of the jaws, which are characterized by the presence of an abnormal overjet. Their relevance becomes evident when their manifestations are severe, as they generate morphologic alterations that bring functional, aesthetic and psychological consequences both in children and adults. The quantitative evaluation of the sagital intermaxillary relationships is done preferably with cephalometry, through measurements that represent diagnostic indicators. There are numerous indices used for intermaxillary sagital classification, nevertheless, the information about their diagnostic value is limited, in terms of the sensibility and specificity associated with their use. The aim of the present narrative review is to analyze the performance of the cephalometric evaluation as a diagnostic tool for the measurement of the intermaxllary sagittal relationship in terms of sensibility and specificity. At present, no single cephalometric test assembles all the ideal characteristics to determine accurately if an individual presents a sagittal discrepancy. The available information related to the properties of specificity and sensitivity of the sagital intermaxillary cephalometric measurements as individual diagnostic indices is scarce. Among them, the ANB angle constitutes a suitable, simple and valid tool to determine these disharmonies. The use of cephalometric diagnostic tests for the sagittal intermaxillary evaluation in growing individuals must consider the dynamic nature of craniofacial development.
\end{abstract}

Rev. Clin. Periodoncia Implantol. Rehabil. Oral Vol. 6(2); 96-101, 2013.

Key words: Cephalometry, intermaxillary sagittal diagnosis, sensitivity, specificity.

\section{INTRODUCCIÓN}

Las maloclusiones en sentido sagital son altamente prevalentes tanto en Chile como en el resto del mundo. Según estudios realizados el año 2007 , la prevalencia de anomalías dentomaxilares en niños de 6 años en Chile es de un $38.29 \%^{(1)}$, cifra que aumenta a los 12 años a un $53 \%{ }^{(2)}$. Dentro de la población preadolescente norteamericana, el $66.4 \%$ posee una relación sagital normal de las bases maxilares, un $31.6 \%$ presenta situaciones de retro-posición mandibular y un $2 \%$ sufre de prognatismo mandibular ${ }^{(3)}$. Otros autores han descrito que la prevalencia de las maloclusiones esqueletales por exceso mandibular en la población caucásica es de un 1 a un $5 \%$, mientras que en la población china y japonesa puede alcanzar un $14 \%(4)$.

Conceptualmente, la evaluación de las relaciones sagitales intermaxilares es un problema de alta relevancia clínica. Lo anterior radica en que cuando sus manifestaciones son severas, éstas generan alteraciones morfológicas con serias consecuencias funcionales, estéticas y psicológicas. En efecto, tanto los individuos portadores de

1. Cirujano Dentista. Licenciado en Odontología. Departamento de Ortodoncia, Universidad de los Andes. Chile.

2. Cirujano Dentista. Cirujano Dentista. Ortodoncista. Licenciado en Odontología. Master en Ciencias. Departamento de Ortodoncia, Universidad de los Andes. Chile.

Correspondencia autor: Rodrigo Oyonarte Weldt. royonarte@miuandes.cl. Trabajo recibido el 23/04/2012. Aprobado para su publicación el 06/05/2012. 
mordida invertida como de un resalte excesivo son evaluados como menos atractivos e inteligentes, y más introvertidos ${ }^{(5)}$. Se ha reportado a su vez que los pacientes que buscan tratamiento ortodóncico no sólo están interesados en corregir su salud y función oral, sino también en mejorar su apariencia y aceptación social(6). Existe una correlación significativa entre la necesidad de tratamiento ortodóncico y calidad de vida relacionada a salud bucal, y entre autoestima y calidad de vida relacionada a salud bucal(6).

Las maloclusiones a su vez tienen alta relevancia funcional, dentro de las cuales destacan las de tipo sagital, expresadas como un resalte excesivo o bien la presencia de mordidas invertidas. Esto supone un desafío terapéutico no sólo en ortodoncia, sino en cualquier campo de la odontología, y especialmente de la rehabilitación oral, donde los objetivos terapéuticos incluyan la corrección de las relaciones de resalte y sobremordida para el logro de relaciones funcionales y estéticas en el sector anterior ${ }^{(7)}$.

Es sabido que el crecimiento del maxilar se manifiesta de manera diferente respecto de la mandíbula, caracterizada por un desarrollo sagital y vertical más tardío(8,9). El proceso de desarrollo y maduración facial progresa con alta intensidad hasta el final de la segunda década de vida, y no cesa por completo hasta la muerte del paciente ${ }^{(9)}$. La implicancia clínica de mayor relevancia tras este concepto es que la relación sagital intermaxilar variará gradualmente entre una edad temprana, donde las diferencias entre individuos normales y anormales son leves, y la vida adulta. En esta última las diferencias son evidentes, pronunciándose la proyección relativa de la mandíbula respecto del maxilar superior. La cantidad de crecimiento proporcional que la mandíbula expresa en relación al maxilar superior define la manifestación de la morfología facial adulta, que es la que en definitiva permite clasificar si un paciente presenta una relación sagital máxilomandibular normal o alterada.

La relación anteroposterior del maxilar con la mandíbula puede ser evaluada a través de la observación clínica, en un proceso subjetivo que permite el abordaje diagnóstico inicial del paciente. Su valoración objetiva normalmente se realiza utilizando la cefalometría en una telerradiografía de perfil(10), usando mediciones cefalométricas. Estas mediciones pueden ser tanto angulares como lineales. Las segundas son sensibles a la magnificación de la placa radiográfica, por lo que la utilización de normas en dicho sentido debe realizarse con cautela. Esto es particularmente cierto luego del advenimiento de la radiología digital, la que en ciertos casos reduce ostensiblemente la magnificación en la exposición radiográfica, afectando la utilidad de dichas medidas cefalométricas lineales.

Los indicadores diagnósticos se utilizan para determinar la presencia o ausencia de un signo clínico o enfermedad. Este indicador permite diferenciar entre dos condiciones, como por ejemplo, saludenfermedad. La capacidad de un indicador como test diagnóstico es medida en términos de sensibilidad y especificidad. La sensibilidad es la capacidad del test para detectar la enfermedad cuando está presente, y la especificidad, la capacidad del test para descartar la enfermedad cuando está ausente ${ }^{(11,12)}$. Es inusual que un test sensible no identifique la presencia de enfermedad, y que un test específico clasifique erróneamente a una persona sana. Habitualmente una prueba diagnóstica posee mejor rendimiento ya sea en su sensibilidad o en su especificidad, siendo una interdependiente de la otra ${ }^{(13)}$. Para diferenciar entre salud y enfermedad, es necesario establecer un valor límite en el cual se base el diagnóstico, para lo cual se utilizan las curvas de rendimiento diagnóstico (Curvas ROC, del inglés "receiver operator characteristic" curve). En un diagnóstico ortodóncico, sin embargo, se establecen rangos de normalidad y de variación biológica, y no simplemente "enfermedad" versus "ausencia de enfermedad"(14), debido a que la pregunta en el diagnóstico ortodóncico habitualmente no es un "si" o "no" absoluto(15).

El uso de las Curvas ROC es habitual en la determinación de la habilidad diagnóstica de un test, ya que muestran los límites de la capacidad de una herramienta para discriminar con exactitud entre dos estados de salud(14). Estas curvas constituyen una herramienta fundamental en la evaluación y comparación de las pruebas diagnósticas ${ }^{(11)}$, y permiten establecer un punto de corte que divide los resultados de la prueba en dos grupos, positivo y negativo, con lo que es posible calcular la sensibilidad y especificidad ${ }^{(11,12)}$. La curva $R O C$ se construye graficando la sensibilidad (verdaderos positivos esperados) en relación a los falsos positivos esperados (1-especificidad)(13). En dicho gráfico se observan todos los pares sensibilidad/especificidad resultantes de la variación continua de los puntos de corte en todo el rango de resultados observados. Entre más arriba y hacia la izquierda esté la curva dentro del diagrama, mejor es la prueba diagnóstica ${ }^{(14)}$, indicando mayores niveles de sensibilidad y especificidad. La prueba diagnóstica ideal tendrá una sensibilidad y una especificidad de un $100 \%{ }^{(11,12)}$, sin embargo, siempre hay una compensación entre éstas. A medida que una aumenta, la otra tiende a disminuir(16).

El área bajo la curva ROC es una medida global de la exactitud de una prueba diagnóstica. Se define como la probabilidad de clasificar correctamente un par de individuos sano y enfermo, seleccionados al azar de la población, mediante los resultados obtenidos al aplicarles la prueba diagnóstica ${ }^{(11)}$. Ésta provee una valoración cuantitativa del desempeño de la prueba y permite comparar la habilidad diagnóstica de distintos tests. Un test es perfecto con un área bajo la curva de 1 , es ideal sobre 0.9 y es apropiado con un área entre 0.7 y 0.9 . Un área menor o igual a 0.5 indica que el test se desempeña no mejor que por azar. Un punto de corte en la curva ROC es el valor para cada análisis cefalométrico donde una clasificación esqueletal cambia a otra ${ }^{(11)}$.

La relación sagital intermaxilar puede ser de Clase I, cuando el maxilar y la mandíbula están en una relación normal, y generalmente se asocia a un perfil recto. Una relación de Clase II se observa cuando la mandíbula se encuentra distal con respecto al maxilar en sentido sagital, cuando hay prognatismo del maxilar o cuando existe una combinación de ambas condiciones. Generalmente se asocia a un perfil convexo. La relación sagital de Clase III se da en el caso de una relación prognática de la mandíbula con respecto al maxilar, cuando éste está retruido o cuando se observa una combinación de ambos estados, y se asocia a un perfil cóncavo. Existen numerosos índices para la clasificación sagital intermaxilar. Sin embargo, es escasa la información respecto de su valor diagnóstico, en términos de la sensibilidad y especificidad asociadas al uso de ellos. La presente revisión narrativa tiene por objetivo analizar el rendimiento de la evaluación cefalométrica como herramienta diagnóstica para la medición de la relación sagital intermaxilar en términos de sensibilidad y especificidad.

\section{METODOLOGÍA}

Se realizaron búsquedas bibliográficas utilizando las bases de datos de Pubmed, Medline y Cochrane Collaboration. Se usaron además las referencias de artículos en revistas especializadas, así como libros texto de ortodoncia y desarrollo cráneo-facial.

Los términos de búsqueda utilizados incluyeron "orthodontic diagnosis", "cephalometric" y "sensitivity and specificity", conectándolos utilizando conectores booleanos ("AND", "OR", "NOT").

Se incluyeron estudios realizados en seres humanos que evaluaran el desarrollo longitudinal de la relación anteroposterior máxilomandibular. También se incluyeron estudios que evaluaran las características de indicadores cefalométricos anteroposteriores en términos de su desempeño como pruebas diagnósticas. Debido a la escasez de literatura en el tema investigado, no se discriminó según tipo de estudio, sino sólo según temática. Así, se incluyeron estudios realizados en humanos tanto transversales como longitudinales, de casos y controles o bien de cohorte histórico.

Al combinar las tres búsquedas y filtrar sólo los documentos en inglés y realizados en humanos, se generaron 39 aciertos de los cuales sólo 8 eran de utilidad para el estudio. Luego se prosiguió con una segunda búsqueda con los términos "cephalometric growth prediction", "cephalometric diagnosis" y "ROC curve", obteniéndose 6 títulos de interés. La búsqueda en colaboración Cochrane no arrojó estudios de utilidad para el presente trabajo.

\section{REVISIÓN}

Entre los numerosos indicadores cefalométricos disponibles para la evaluación de la relación sagital intermaxilar, son pocos los que han sido evaluados en base a sus propiedades como herramienta diagnóstica. Éstos incluyen el ángulo ANB, descrito entre los puntos $A$, Nasion y $\mathrm{B}^{(17)}$, la diferencia de unidades mandibular-maxilar de Harvold(18), 
la apreciación o análisis de Wits ${ }^{(19,20)}$, la distancia anteroposterior entre los puntos $A$ y B proyectados sobre el plano de Frankfort (AF-BF) ${ }^{(21)}$ y el índice de displasia anteroposterior o APDI, por sus siglas en inglés ${ }^{(22)}$.

Oktay ${ }^{(23)}$ llevó a cabo un estudio transversal observacional analítico en el cual realizó un análisis de correlación sobre distintas medidas cefalométricas verticales y sagitales en telerradiografías de 145 pacientes de ambos sexos de entre 9 y 14 años, incluyendo las siguientes medidas: ángulo ANB, análisis de Wits, AF-BF y APDI. Las cuatro mediciones sagitales se encontraron significativamente correlacionadas $(p<0.01)$. El ángulo ANB es el menos afectado por variables verticales, haciéndolo un buen indicador diagnóstico de las displasias sagitales intermaxilares. Dicho autor concluyó que el ángulo ANB no era menos confiable que cualquier otra medida cefalométrica como un parámetro sagital anteroposterior.

Por su parte, Hurmerinta y cols(24) compararon el ángulo ANB, el análisis de Wits y la percepción visual para clasificar las relaciones sagitales de la mandíbula. Dicho estudio transversal fue realizado en 497 niños de entre 4 y 20 años, y concluyó que el ángulo ANB disminuía en promedio desde $3.8^{\circ}$ entre los 4 y 9 años a $3.0^{\circ}$ entre los 12 y 20 años, y que había mayor concordancia entre el ángulo ANB y la percepción visual, que entre el análisis de Wits y dicha percepción. Este estudio sugiere considerar la apreciación clínica para discriminar respecto de la clasificación anteroposterior intermaxilar en casos de mayor complejidad.

Han y $\mathrm{Kim}^{(25)}$ realizaron un estudio transversal de casos y controles para evaluar la habilidad diagnóstica de distintas medidas cefalométricas en la identificación de alteraciones Clase II y III esqueletal. Éste se llevó a cabo en 967 pacientes de 8 a 17 años; 624 pacientes presentaban Clase II y 36 Clase III. Los individuos fueron clasificados según su relación molar en maloclusión Clase I, II ó III. Se evaluaron 15 medidas cefalométricas, y se generaron curvas ROC con el software "ROC Analyzer". Entre numerosos análisis anteroposteriores, APDI fue el de mejor desempeño diagnóstico. ANB, sin embargo, tuvo un desempeño apropiado. Los resultados de los análisis de curvas ROC para este estudio son presentados en las Tablas 1 y 2.

Otro grupo de investigadores ${ }^{(14)}$ realizó un estudio comparativo del desempeño de cinco variables de evaluación sagital intermaxilar, utilizando curvas ROC. Las telerradiografías de perfil de 155 individuos entre 10 y 18 años fueron clasificadas por expertos en base a la apreciación clínica del perfil y de su oclusión en maloclusiones Clase I, II y III, utilizando dicho criterio como gold standard. Se desarrollaron normas cefalométricas de clasificación utilizando curvas ROC y se comparó el desempeño de los indicadores cefalométricos utilizando normas convencionales y aquellas derivadas del análisis con curvas ROC. Al evaluar el desempeño del ángulo ANB con metodología ROC, el área bajo la curva obtenida para la diferenciación de Clases I y II fue de 0.954, mientras que para la diferenciación de Clases I y III fue de 0.934 . Los resultados fueron similares a los obtenidos con otros instrumentos diagnósticos estudiados siendo el ANB uno de los mejor evaluados. Las cinco variables de evaluación sagital intermaxilar utilizadas incluyeron el ángulo ANB, análisis de McNamara, diferencia de unidades de Harvold, APDI y apreciación de Wits. En el caso de las dos primeras, su desempeño con ambos tipos de normas era equivalente, el cual mejoró para las otras variables al utilizar las normas derivadas del uso de curvas ROC. Todas resultaron ser apropiadas para el diagnóstico de relaciones sagitales intermaxilares. Los resultados de este estudio se presentan en la Tabla 3.

Para realizar el diagnóstico sagital intermaxilar, es importante considerar que los huesos maxilares se caracterizan por presentar un desarrollo diferencial. Esto ha sido reportado por numerosos autores ${ }^{(24-30)}$. Utilizando una muestra longitudinal, Ochoa y $\mathrm{Nanda}^{(30)}$ evaluaron 28 individuos de ambos sexos y observaron que el ángulo ANB disminuye significativamente durante el crecimiento. Dicha disminución frecuentemente se atribuye a la reducción de la distancia sagital máxilomandibular relacionada con la edad, dada por una dominancia del crecimiento mandibular cuando se compara con el crecimiento maxilar sagital( ${ }^{(31)}$. La reducción del ángulo ANB con la edad también podría deberse en parte a la rotación antihoraria de los planos oclusal y mandibular ${ }^{(26)}$

Van Diepenbeek y cols ${ }^{(29)}$ utilizaron registros longitudinales de 449 pacientes de tres centros de crecimiento distintos. Se observó que entre los 9 y 14 años tanto los ángulos SNA como SNB aumentaban, mientras ANB disminuía. Lux y cols ${ }^{(31)}$ determinaron que la disminución del ángulo ANB era estadísticamente significativa entre los 7 y los 15 años. En su trabajo, la reducción del ANB fue mayor en niños que en niñas $\left(1.65^{\circ}\right.$ versus $\left.1.3^{\circ}\right)$. Estos investigadores concluyeron que en sujetos con buena oclusión y Clase I, se observa una reducción de la distancia sagital máxilomandibular durante el desarrollo prepuberal y puberal como resultado de una relativa dominancia del crecimiento sagital mandibular.

La Tabla 4 resume los hallazgos encontrados de la revisión bibliográfica con respecto a la disminución del ANB a lo largo del crecimiento.

Tabla 1. Análisis ROC con método paramétrico para la determinación de maloclusiones Clase II.

\begin{tabular}{|c|c|}
\hline Medidas cefalométricas & Áreas y errores estándar \\
\hline APDI & $0.829(\mathrm{e}=0.014)$ \\
\hline Apreciación Wits & $0.812(\mathrm{e}=0.015)$ \\
\hline Overjet & $0.805(\mathrm{e}=0.015)$ \\
\hline Ángulo del plano AB & $0.774(\mathrm{e}=0.016)$ \\
\hline ANB & $0.752(\mathrm{e}=0.017)$ \\
\hline Convexidad & $0.722(\mathrm{e}=0.017)$ \\
\hline SNB & $0.645(\mathrm{e}=0.019)$ \\
\hline SN- Pog & $0.622(\mathrm{e}=0.020)$ \\
\hline Ángulo Facial & $0.621(\mathrm{e}=0.020)$ \\
\hline SNA & $0.576(\mathrm{e}=0.051)$ \\
\hline Eje- $\mathrm{Y}$ & $0.548(\mathrm{e}=0.020)$ \\
\hline Ángulo Goniaco & $0.543(\mathrm{e}=0.020)$ \\
\hline PP- MP & $0.539(\mathrm{e}=0.020)$ \\
\hline MP- SN & $0.486(\mathrm{e}=0.020)$ \\
\hline MP- FH & $0.464(\mathrm{e}=0.020)$ \\
\hline
\end{tabular}

Tomado de Han U, Kim Y. Determination of Class II and Class III skeletal patterns: receiver operating characteristic (ROC) analysis on various cephalometric measurements. Am J Orthod Dentofacia Orthop. 1998;113(5):538-545

Tabla 2. Análisis ROC con método paramétrico para la determinación de maloclusiones Clase III.

\begin{tabular}{|c|c|}
\hline Medidas cefalométricas & Áreas y errores estándar \\
\hline APDI & $0.937(e=0.018)$ \\
\hline Apreciación Witts & $0.931(e=0.018)$ \\
\hline Convexidad & $0.925(e=0.020)$ \\
\hline Ángulo del plano $A B$ & $0.920(e=0.022)$ \\
\hline ANB & $0.912(e=0.022)$ \\
\hline Overjet & $0.897(e=0.028)$ \\
\hline Eje- $Y$ & $0.789(e=0.045)$ \\
\hline SNB & $0.754(e=0.042)$ \\
\hline Ángulo Facial & $0.751(e=0.046)$ \\
\hline SN- Pog & $0.717(e=0.050)$ \\
\hline Ángulo Gonial & $0.665(e=0.048)$ \\
\hline SNA & $0.576(e=0.051)$ \\
\hline PP- MP & $0.476(e=0.050)$ \\
\hline MP-SN & $0.441(e=0.049)$ \\
\hline MP- FH & $0.392(e=0.053)$ \\
\hline
\end{tabular}

Tomado de Han U, Kim Y. Determination of Class II and Class III skeletal patterns: receiver operating characteristic (ROC) analysis on various cephalometric measurements. Am J Orthod Dentofacial Orthop. 1998;113(5):538-545. 


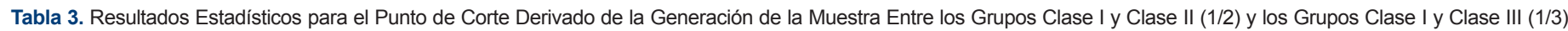
y Aplicado a la Conformación de la Muestra $(a, b)$

\begin{tabular}{|c|c|c|c|c|c|c|c|c|c|}
\hline Medida & Decisión & Punto de Corte & $\begin{array}{l}\text { Diagnóstico } \\
\text { Exitoso }\end{array}$ & $\%$ de Acuerdo & $\begin{array}{c}\% \text { de Acuerdo } \\
\text { IC }, 95\end{array}$ & Kappa & Kappa IC ,95 & $\mathrm{AUC} a$ & AUC IC ,95 \\
\hline \multirow{2}{*}{ ANB } & $1 / 2$ & 3.6 & $45 / 51$ & 88.2 & $76.1 / 95.6$ & 0.59 & $0.59 / 0.94$ & 95.4 & $89.1 / 100$ \\
\hline & $1 / 3$ & 0.1 & $47 / 51$ & 92.2 & $81.1 / 97.8$ & 0.84 & $0.69 / 0.99$ & 93.4 & $85.8 / 100$ \\
\hline \multirow{2}{*}{ мстот } & $1 / 2$ & 2.8 & $44 / 51$ & 86.3 & $73.7 / 94.3$ & 0.72 & $0.53 / 0.91$ & 92.1 & $83.8 / 100$ \\
\hline & $1 / 3$ & -3.3 & $46 / 51$ & 90.2 & $81.1 / 97.8$ & 0.80 & $0.64 / 0.97$ & 94.0 & $86.8 / 100$ \\
\hline \multirow{2}{*}{ WITS } & $1 / 2$ & 2.1 & $48 / 51$ & 94.1 & $83.8 / 98.8$ & 0.88 & $0.75 / 1.0$ & 98.3 & $94.4 / 100$ \\
\hline & $1 / 3$ & -4.0 & $47 / 51$ & 92.2 & $78.6 / 96.7$ & 0.84 & $0.70 / 0.99$ & 96.7 & $91.3 / 100$ \\
\hline \multirow{2}{*}{ APDI } & $1 / 2$ & 83.6 & $43 / 51$ & 84.3 & $71.4 / 93.0$ & 0.69 & $0.48 / 0.89$ & 88.7 & $78.8 / 100$ \\
\hline & $1 / 3$ & 97.9 & $44 / 51$ & 86.3 & $73.7 / 94.3$ & 0.73 & $0.54 / 0.91$ & 90.0 & $80.7 / 99.3$ \\
\hline \multirow{2}{*}{ UNITDIF } & $1 / 2$ & 26.9 & $44 / 51$ & 86.3 & $73.7 / 94.3$ & 0.72 & $0.53 / 0.91$ & 92.8 & $84.9 / 100$ \\
\hline & $1 / 3$ & 32.7 & $40 / 51$ & 78.4 & $64.7 / 88.7$ & 0.57 & $0.34 / 0.79$ & 86.1 & $75.2 / 97.0$ \\
\hline
\end{tabular}

(a) AUC indica el área bajo la curva ROC; APDI, índice de displasia anteroposterior.

(b) Diagnóstico exitoso indica el acuerdo entre el valor cefalométrico usando el indicado punto de corte y la clasificación del gold estándar.

Tomado de Anderson G, Fields H, Beck M, Chacon G, Vig K. Development of cephalometric norms using a unified facial and dental approach. Angle Orthod, 2006 Jul; 76(4): 612-618.

Tabla 4. Disminución del ángulo ANB a lo largo del crecimiento.

\begin{tabular}{|c|c|c|c|c|c|c|c|c|c|c|c|c|c|c|c|c|c|c|c|c|}
\hline \multicolumn{21}{|c|}{ Clase I } \\
\hline \multirow{2}{*}{ Autores } & \multirow{2}{*}{$\mathrm{N}$} & \multirow{2}{*}{ Muestra } & \multicolumn{2}{|c|}{9 años } & \multicolumn{2}{|c|}{11 años } & \multicolumn{2}{|c|}{12 años } & \multicolumn{2}{|c|}{13 años } & \multicolumn{2}{|c|}{14 años } & \multicolumn{2}{|c|}{15 años } & \multicolumn{2}{|c|}{16 años } & \multicolumn{2}{|c|}{17 años } & \multicolumn{2}{|c|}{19 años } \\
\hline & & & $x$ & DS & $x$ & DS & $x$ & DS & $x$ & DS & $x$ & DS & $x$ & DS & $x$ & DS & $x$ & DS & $x$ & DS \\
\hline Hamamci y cols. 2006 (41) & 28 & Hy $\mathrm{M}$ & 3.71 & 0.96 & & & & & & & 3.00 & 1.06 & & & & & & & 2.57 & 1.01 \\
\hline Palleck y cols. 2001 (28) & 39 & Hy $\mathrm{M}$ & & & & & 2.54 & 1.17 & & & 2.13 & 1.47 & & & 2.02 & 1.52 & & & & \\
\hline Bishara y cols. 1983 (26) & 20 & $\mathrm{H}$ & 3.50 & 1.60 & & & 3.80 & 1.70 & & & 3.60 & 1.90 & & & 3.20 & 1.90 & 3.00 & 2.00 & & \\
\hline Bishara y cols. 1983 (26) & 15 & M & 4.00 & 1.60 & & & 3.60 & 1.90 & & & 3.10 & 2.10 & & & 3.00 & 2.10 & 3.20 & 2.10 & & \\
\hline Baccetti y cols. 2009 (42) & 30 & $\mathrm{HyM}$ & & & & & & & & & & & & & 1.30 & 1.50 & & & 1.30 & 1.50 \\
\hline $\begin{array}{l}\text { Lux y cols. } \\
2005 \text { (31) }\end{array}$ & 19 & $\mathrm{H}$ & 3.41 & 2.01 & 3.20 & 1.97 & & & 3.02 & 2.06 & & & & 2.45 & & 2.33 & & & & \\
\hline $\begin{array}{l}\text { Lux y cols. } \\
2005 \text { (31) }\end{array}$ & 18 & M & 3.09 & 2.28 & 2.91 & 2.67 & & & 2.45 & 2.72 & & & & 2.40 & & 2.96 & & & & \\
\hline \multicolumn{21}{|c|}{ Clase II } \\
\hline Baccetti y cols. 2009 (42) & 23 & H y M & & & & & & & & & & & & & 5.3 & 1.10 & & & 5.30 & 1.30 \\
\hline $\begin{array}{l}\text { Lux y cols. } \\
2005 \text { (31) }\end{array}$ & 8 & $\mathrm{H}$ & 5.45 & 1.97 & 5.25 & 1.64 & & & 5.60 & 1.59 & & & & 5.97 & & 1.73 & & & & \\
\hline $\begin{array}{l}\text { Lux y cols. } \\
2005 \text { (31) }\end{array}$ & 9 & M & 4.56 & 1.38 & 4.58 & 1.97 & & & 4.83 & 2.46 & & & & 4.48 & & 2.35 & & & & \\
\hline Foley y cols. 1997 (27) & 15 & $\mathrm{H}$ y M & & & & & 5.30 & 2.14 & & & 4.90 & 2.01 & & & 4.77 & 2.37 & & & & \\
\hline \multicolumn{21}{|c|}{ Clase III } \\
\hline Palleck y cols. 2001 (28) & 9 & $\mathrm{H}$ y M & & & & & 0.91 & 1.57 & & & 1.04 & 1.8 & & & 0.2 & 1.22 & & & & \\
\hline Baccetti y cols. 2005 (43) & $32-65$ & $\mathrm{H}$ & 0.70 & 2.2 & 0.60 & 2.20 & 0.10 & 2.50 & 0.80 & 2.4 & 0.40 & 2.40 & 0.20 & 2.80 & 0.30 & 3.20 & 1.30 & 2.80 & & \\
\hline Baccetti y cols. 2005 (43) & $31-65$ & M & 0.50 & 2.40 & 0.10 & 2.00 & 0.50 & 2.50 & -0.20 & 2.70 & -0.10 & 2.00 & -0.90 & 2.80 & -1.10 & 2.60 & -0.50 & 3.10 & & \\
\hline Baccetti y cols. 2009 (42) & 22 & H y M & -0.90 & 2.10 & & & & & & & & & -2.10 & 2.8 & & & & & & \\
\hline
\end{tabular}




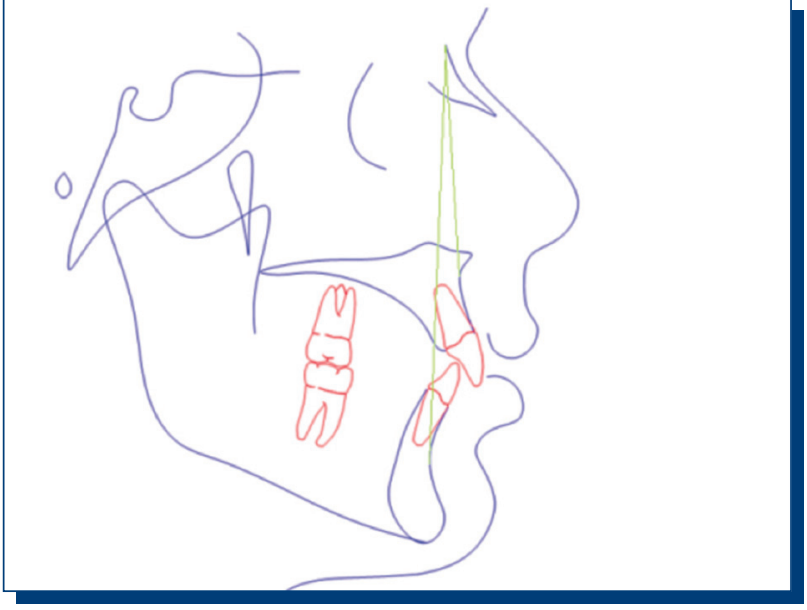

Figura 1. Ángulo ANB.

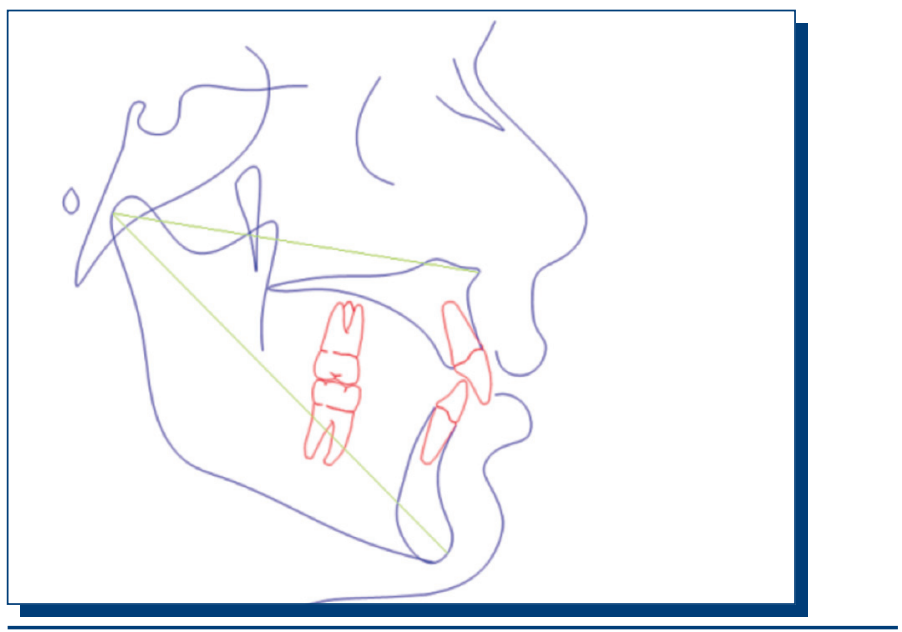

Figura 2. Diferencia de unidades mandibular-maxilar de Harvold.

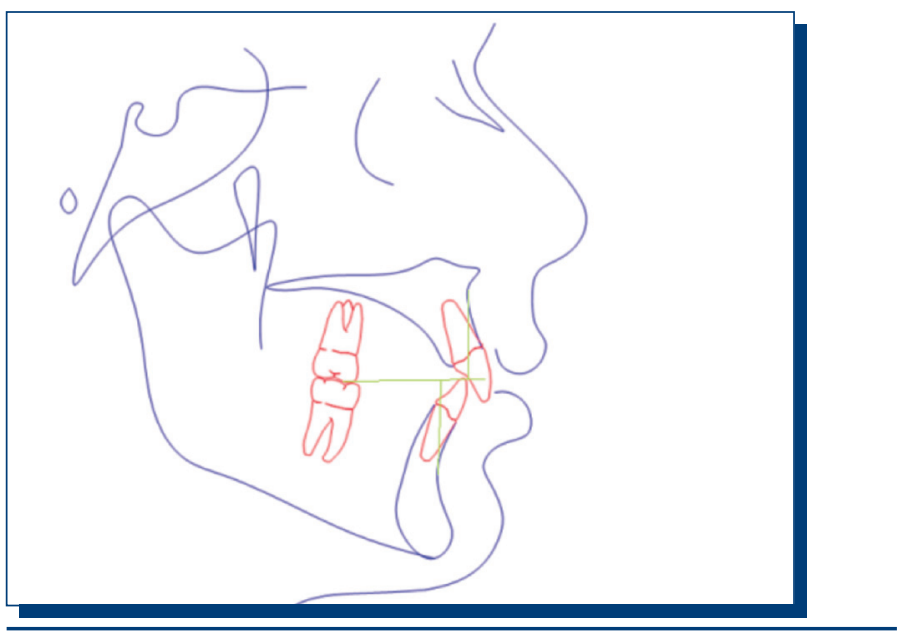

Figura 3. Análisis de Wits.

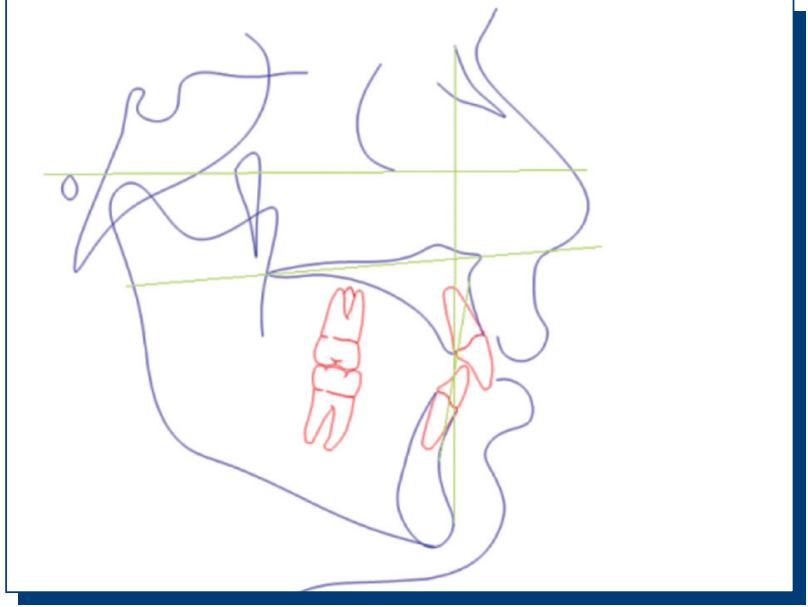

Figura 4. Análisis de displasia anteroposterior (APDI).

\section{DISCUSIÓN}

La evaluación de la relación sagital intermaxilar es realizada utilizando diferentes indicadores cefalométricos. En base a las diferencias observadas en términos de la especificidad y sensibilidad de estos instrumentos diagnósticos, éstos podrían entregar resultados discordantes entre sí cuando un mismo paciente es diagnosticado utilizando dos o más análisis cefalométricos distintos. Lo anterior puede generar confusión respecto de las conductas clínicas a seguir, especialmente en pacientes con características límite entre dos clasificaciones sagitales ${ }^{(32)}$. Esto ocurre debido a que no existe una única prueba aceptada que determine la presencia o la severidad de la displasia esqueletal anteroposterior ${ }^{(32)}$. A pesar de ello, y de que se han reportado debilidades en relación a su desempeño en pacientes con variaciones en longitud e inclinación de la base de cráneo(19) y de los maxilares ${ }^{(33)}$, el ángulo ANB se constituye como la medida cefalométrica más comúnmente utilizada para diagnosticar las displasias sagitales esqueletales ${ }^{(10,19,26,34-36)}$. En efecto, el uso del ángulo ANB es generalizado entre los ortodoncistas debido a que es de fácil medición, y a que sus resultados son generalmente consistentes con la presentación clínica en la mayoría de los casos. La confiabilidad de esta medida(17) se reafirma a través de su buen desempeño al evaluarse como prueba diagnóstica ${ }^{(10,19,26,34-36)}$. Al valorar distintas medidas cefalométricas como herramienta diagnóstica, el ángulo ANB representa un test diagnóstico de alta especificidad y sensibilidad en la evaluación sagital intermaxilar. Provee así un punto de referencia confiable a partir del cual trabajar que es familiar para la mayoría de los clínicos ${ }^{(14,23,26-28)}$.

A pesar de que el ángulo ANB es ampliamente utilizado en odontología, habitualmente se usa considerando un valor promedio de $2^{\circ}$ y una variación de la normalidad de más/menos $2^{\circ(37)}$. Dicho abordaje diagnóstico no toma en cuenta la edad ni los fenómenos de crecimiento y desarrollo esperados para un determinado individuo, que se reflejan en la reducción del ángulo ANB a lo largo del desarrollo(24,26,29,31,38,39). Esto puede llevar a un diagnóstico errado, y por ende a resultados clínicos distintos de los esperados.

Como puede desprenderse de esta revisión, la información disponible respecto de las pruebas cefalométricas sagitales intermaxilares es muy escasa y con diseños experimentales débiles. Se requieren más estudios sobre la validez y rendimiento diagnóstico de las diferentes medidas. Las conclusiones sobre la relación sagital intermaxilar deben idealmente basarse en una combinación de métodos diagnósticos en lugar de en una sola medición, como lo son el ángulo ANB, el análisis de Wits, el APDI y otros ${ }^{(19,26,40)}$. Lo anterior se enmarca dentro de las recomendaciones emanadas por Jacobson ${ }^{(20)}$ y Bishara ${ }^{(26)}$, que recomiendan el uso combinado de análisis de Wits y la medición del ANB. Del mismo modo, Ishikawa y cols ${ }^{(40)}$ sugieren una combinación de ANB, Wits y APDI como un método clínicamente apropiado para la evaluación de las relaciones de la mandíbula en los individuos. 


\section{CONCLUSIONES}

En la actualidad no existe una sola prueba de características ideales para determinar con precisión si un individuo presenta una Clase I, II ó III esqueletal, siendo a su vez escasa la información actualmente disponible referente a las propiedades de especificidad y sensibilidad de las mediciones cefalométricas sagitales intermaxilares.

Entre los indicadores diagnósticos cefalométricos disponibles, el ángulo ANB constituye una herramienta simple y válida para determinar estas desarmonías que debe ser utilizada idealmente en conjunto con una o más medidas cefalométricas sagitales.

\section{CONFLICTO DE INTERESES}

Los autores declaran no tener conflictos de interés.

\section{REFERENCIAS BIBLIOGRÁFICAS}

1. Soto L, Tapia R. Diagnóstico Nacional de Salud Bucal de los niños de 6 años. In: MINSAL, editor. Chile 2007.

2. Soto L, Tapia R. Diagnóstico Nacional de Salud Bucal del Adolescente de 12 años y Evaluación del Grado de Cumplimiento de los Objetivos Sanitarios de Salud Bucal 2000-2010. Chile 2007.

3. Popovich F. A review of studies from the burlington Growth Centre, 1956-1989, en essays in honor of Robert E Moyers. Hunter WS, Carlson DS (Eds). Craniofacial Growth Series, 24: 313-344. Center for Human Growth and Development. The University of Michigan. Ann Arbor, 1991.

4. Cozza P, Marino A, Mucedero M. An orthopaedic approach to the treatment of Class III malocclusions in the early mixed dentition. Eur J Orthod, 2004; 26(2): 191 199.

5. Olsen JA, Inglehart MR. Malocclusions and perceptions of attractiveness, intelligence, and personality, and behavioural intentions. AJODO, 2011; 140: 669679.

6. Kiyak HA. Does orthodontic treatment affect patients' quality of life? J Dent Educ, 2008 Aug; 72(8): 886-894.

7. De Baets E, Lambrechts H, Lemiere J, Diya L, Willems G. Impact of self-esteem on the relationship between orthodontic treatment need and oral health-related quality of life in 11- to 16-year-old children. Eur J Orthod, 2012 Dec; 34(6): 731737.

8. Enlow D, Hans M. Essentials of facial growth. Philadelphia, WB Saunders Co., 1996.

9. Behrents R. Growth in the aging skeleton. Monograph 17 Craniofacial Growth Series. Center for Human Growth and Development. The University of Michigan, Ann Arbor, Michigan 1985.

10. Freeman R. Adjusting A-N-B angles to reflect the effect of maxillary position. Angle Orthod, 1981; 51(2): 162-171.

11. Burgueno M, Garcia-Bastos J, Gonzalez-Buitrago J. ROC curves in the evaluation of diagnostic tests. Med Clin (Barc), 1995 May 6; 104(17): 661670.

12. McGorray S. Sensitivity, specificity, and related concepts. Seminars in Orthodontics, 2002; 8(2): 92-101.

13. Fletcher RH, Fletcher SW, Wagner EH. Clinical Epidemiology: The Essentials 3rd ed. Lippincott, Philadelphia (USA),1996.

14. Anderson G, Fields H, Beck M, Chacon G, Vig K. Development of cephalometric norms using a unified facial and dental approach. Angle Orthod, 2006 Jul; 76(4): 612-618.

15. Freudenthaler J, Celar A, Schneider B. Overbite depth and anteroposterior dysplasia indicators: The relationship between occlusal and skeletal patterns using the receiver operating characteristic (ROC) analysis. Eur J Orthod, $2000 \mathrm{Feb} ; 22(1)$ : 75-83.

16. Parfrey P, Barrett B. Clinical epidemiology: Practice and methods. New York, N.Y. London: Humana Press; Springer, distributor; 2009.

17. Riedel R. Esthetics and its relation to orthodontic therapy. Angle Orthod, 1950; 20: $168-178$.

18. Harvold E. The activator in interceptive orthodontics. St. Louis. The C.V. Mosby Company, 1974.

19. Jacobson A. The "Wits" appraisal of jaw disharmony. Am J Orthod, 1975 Feb; 67(2): 125-138.

20. Jacobson A. Application of the "Wits" appraisal. Am J Orthod, 1976; 70: 179189.

21. Chang H. Assessment of anteroposterior jaw relationship. Am J Orthod Dentofac Orthop, 1987; 92: 117-122.

22. Kim Y, Vietas J. Anteroposterior displasia indicator: An adjunt to cephalometric differential diagnosis. Am J Orthod, 1978; 73: 619-633.
23. Oktay H. A comparison of ANB, WITS, AF-BF, and APDI measurements. Am J Orthod Dentofacial Orthop, 1991; 99: 122-128.

24. Hurmerinta K, Rahkamo A, Haavikko K. Comparison between cephalometric classification methods for sagittal jaw relationships. Eur J Oral Sci, 1997 Jun; 105(3): 221-227.

25. Han U, Kim Y. Determination of Class II and Class III skeletal patterns: Receiver operating characteristic (ROC) analysis on various cephalometric measurements Am J Orthod Dentofacial Orthop, 1998; 113(5): 538-545.

26. Bishara S, Fahl J, Peterson L. Longitudinal changes in the ANB angle and Wits appraisal: Clinical implications. Am J Orthod, 1983 Aug; 84(2): 133-139.

27. Foley TF, Stirling DL, Hall-Scott J. The reliability of three sagittal reference planes in the assessment of Class II treatment. Am J Orthod Dentofacial Orthop, 1997 Sep; 112(3): 320-326; discussion 7-9.

28. Palleck S, Foley TF, Hall-Scott J. The reliability of 3 sagittal reference planes in the assessment of Class I and Class III treatment. Am J Orthod Dentofacial Orthop, 2001 Apr; 119(4): 426-435.

29. Van Diepenbeek AF, Buschang PH, Prahl-Andersen B. Age-dependant cephalometric standards as determined by multilevel modeling. Am J Orthod Dentofacial Orthop, 2009 Jan; 135(1): 79-87.

30. Ochoa B, Nanda R. Comparison of maxillary and mandibular growth. Am J Orthod Dentofacial Orthop, 2004; 125(2): 148-159.

31. Lux C, Burden D, Conradt C, Komposch G. Age-related changes in sagital relationship between the maxilla and mandible. Eur J Orthod, 2005; 27: 568 578

32. Bishara S. Ortodoncia. 1 ed: McGraw-Hill/Interamericana; 2001.

33. Iwasaki H, Ishikawa H, Chowdhury L, Nakamura S, Lida J. Properties of the ANB angle and the Wits appraisal in the skeletal estimation of Angle's Class III patients. Eur J Orthod, 2002 Oct; 24(5): 477-483.

34. Ferrazzini G. Critical evaluation of the ANB angle. Am J Orthod, 1976 Jun; 69(6): 620-626.

35. Canut J. Ortodoncia clínica y terapéutica. $2^{a}$ ed. Barcelona: Masson. 2000.

36. Zupancic S, Pohar M, Farcnik F, Ovsenik M. Overjet as a predictor of sagittal skeletal relationships. Eur J Orthod, 2008 Jun; 30(3): 269-273.

37. Steiner C. Cephalometrics for you and me. Am J Orthod Dentofacial Orthop, 1953; 39: 729-755.

38. Nanda RS, Ghosh J. Longitudinal growth changes in the sagittal relationship of maxilla and mandible. Am J Orthod Dentofacial Orthop, 1995 Jan; 107(1): 79-90.

39. Silveira A, Fishman L, Subtelny D, Kassebaum D. Facial growth during adolescence in early, average and late maturers. The Angle Orthodontist, 1992; 62 (3): 185-190.s.

40. Ishikawa H, Nakamura S, Iwasaki H, Kitazawa. Seven parameters describing anteroposterior jaw relationships: Postpubertal prediction accuracy and interchangeability. Am J Orthod Dentofacial Orthop, 2000; 117 : 714-720.

41. Hamamci N, Basaran G, Kiralp S, Sahin S, Selek M, Arslan SG. Longitudinal study of untreated skeletal Class I subjects growth and development with McNamara cephalometric analysis. Biotechnol \& Biotechnol Eq, 2006; 3(20): 175183.

42. Baccetti T, Stahl F, McNamara J. Dentofacial growth changes in subjects with untreated Class II malocclusion from late puberty through young adulthood. Am J Orthod Dentofacial Orthop, 2009; 135(2): 148-154.

43. Baccetti T, Reyes BC, McNamara JA. Gender differences in Class II maloclussion. Angle Orthod, 2005; 75(4): 510-520. 